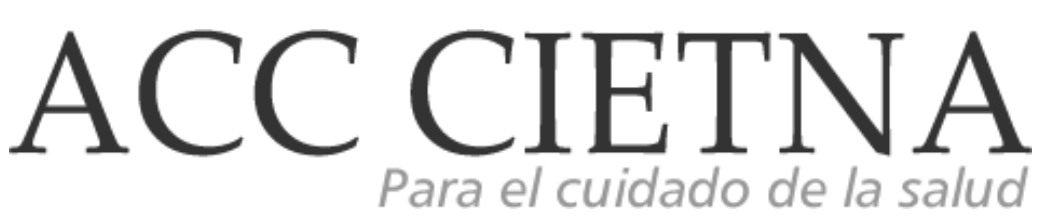

\title{
Teoría fundamentada como referente metodológico en el cuidado de enfermería
}

\section{Grounded theory as a methodological reference in nursing care}

\author{
Claudia Alejandra Cervantes-Lara *, 1, b, d \\ claudiacervantes43548@gmail.com \\ https://orcid.org/0000-0003-0157-5423
}

Leticia Casique-Casique ${ }^{1,}$ a, c

leticiacc_2004@yahoo.com.mx

https://orcid.org/0000-0002-0532-4819

Alicia Álvarez-Aguirre ${ }^{1,}$ a

alicia.alvarez@ugto.mx

https://orcid.org/0000-0001-5538-7634

\section{* Autor corresponsal}

\author{
${ }^{1}$ División de Ciencias de la Salud e ingenierías, Campus \\ Celaya Salvatierra, Universidad de Guanajuato, México \\ a Doctora en Ciencias de Enfermería en enfermería \\ b Maestra en Ciencias de Enfermería \\ c Docente de la División de Ciencias de la Salud e \\ ingenierías
d Estudiante del Programa de Doctorado en Ciencias de Enfermería

\section{Fechas importantes \\ Recibido: 2021-06-29 \\ Aceptado: 2021-11-15}

Publicado online: 2021-12-17

\begin{abstract}
Resumen
Los cambios sociales exigen a un profesional de enfermería capaz de afrontar los retos que la sociedad presenta, por lo que es necesario indagar en los diferentes enfoques que la investigación ofrece para responder a un deber ético-moral, es así que en el presente se narra la utilidad de la Teoría Fundamentada en los Datos utilizada como referente metodológico, cuya aplicación se considera cuando existen fenómenos que han sido poco abordados, falta de teorías formales o una nueva forma de aproximarse a la realidad social, pues coadyuva a que el profesional de enfermería pueda profundizar, comprender e interpretar una amplia diversidad de fenómenos posibilitando la innovación en los conocimientos para mejorar la calidad en los cuidados que proporciona enfermería. Además, se indaga en la aplicación de este referente dentro del área de la sexualidad, tema esencial en el ser humano debido a que está presente en todas las etapas de la vida y el cuidado de esta es fundamental para el bienestar integral de la persona.
\end{abstract}

Palabras clave: Teoría fundamentada, cuidado, enfermería, sexualidad

\section{Abstract}

Social changes require a nursing professional capable of facing the challenges that society presents, so it is necessary to investigate the different approaches that research offers to respond to an ethicalmoral duty, that is how in the present it is narrated the usefulness of the Grounded Theory used as a methodological reference, whose application is considered when there are phenomena that have been little addressed, lack of formal theories or a new way of approaching social reality, since it helps the 
nursing professional can deepen, understand and interpret a wide diversity of phenomena enabling innovation in knowledge to improve the quality of care provided by nursing. Likewise, the application of this reference within the area of sexuality is investigated, an essential issue in the human being because it is present in all stages of life and care in this area is essential for the integral well-being of the person.

Keywords: Grounded theory, care, nursing, sexuality

\section{Introducción}

Enfermería es una disciplina práctica que, a través de la investigación, teorías de rango medio, proyectos de gestión, entre otros, está consolidando su cuerpo de conocimientos. Es a partir de la investigación que se delimita el objeto de conocimiento y se evidencia qué, cómo, por qué y para qué se hace ${ }^{1}$.

Es importante mencionar que la investigación en enfermería se aborda desde tres enfoques: cualitativo, cuantitativo y complementario o mixto, los cuales surgieron para indagar determinados fenómenos e incluso el estudio de lo que nos rodea.

El enfoque cuantitativo representa un proceso secuencial que permite comprobar algunas suposiciones, lleva un orden riguroso y las unidades analizadas se vinculan a mediciones obtenidas de las que se extraen conclusiones acordes a la o a las hipótesis planteadas previamente, dado que los resultados se pretenden generalizar y difundir, pues se basa en la lógica o razonamiento deductivo, es decir, que inicia de lo general para concluir en lo particular.

Por su parte, el enfoque cualitativo se desarrolla del razonamiento inductivo, es decir, que el investigador inicia analizando los hechos y los estudios previos, con el fin de generar una teoría que interprete lo que se observa. Es fundamental señalar que el problema de investigación planteado no es muy específico, esto se va delimitando paulatinamente, además conviene señalar que el proceso es flexible, pues no pretende generalizar los resultados, sino comprender el fenómeno desde la perspectiva de los participantes que lo viven.

Por último, el enfoque mixto enlaza la investigación cualitativa y cuantitativa, por lo que implica la colecta y análisis de datos cuantitativos y cualitativos logrando un mayor entendimiento del fenómeno de estudio².

Cabe mencionar que, de manera más específica, en el enfoque cualitativo se cuenta con la Teoría Fundamentada en los Datos [TFD] utilizada como referente metodológico, cuya aplicación se considera cuando existen fenómenos poco abordados, falta de teorías formales o una nueva forma de aproximarse a la realidad social.

La aplicación de la TFD dentro del área de enfermería es de suma importancia, debido a que la TFD pretende generar interpretaciones que puedan explicar y/o proporcionar información sobre las conductas sometidas a estudio, lo que permite al profesional de enfermería profundizar, comprender e interpretar una amplia diversidad de fenómenos posibilitando planear y ejecutar un mejor cuidado ${ }^{3}$.

Asimismo, es el soporte metodológico que apoya el proceso y conduce a la interpretación de las interacciones humanas en las que se ve inmerso el profesional de enfermería.

Por lo que Oliveira refiere que la TFD es una herramienta cada vez más implementada por el profesional de enfermería en el área asistencial, gerencial, educativo y de formación, lo que ha producido un mayor número de publicaciones científicas bajo este referente metodológico ${ }^{4}$. 
Cabe resaltar que enfermería como ciencia tiene algunas áreas de estudio o campos en investigación que le permiten continuar desarrollándose hacia la excelencia en el cuidado, algunas de estas áreas son la epidemiología, la coordinación de proyectos de investigación, los registros con fines de investigación, la docencia en investigación, la investigación clínica y la investigación en gestión de calidad ${ }^{5}$. Dado que enfermería brinda un cuidado integral en diferentes áreas o campos, es necesario que los profesionales de enfermería estén actualizándose en forma continua para brindar un cuidado de calidad en forma integral, pues al no brindar dicha atención puede generar numerosas problemáticas de índole físico, social, emocional, familiar, entre otras.

\section{Desarrollo}

\section{Teoría Fundamentada en los Datos como referente metodológico}

La TFD se sustenta en la teoría del Interaccionismo Simbólico (IS), cuyo propósito es comprender cómo las personas definen un acontecimiento o fenómeno a través de su interacción social ${ }^{6}$.

Por lo que, la TFD suele utilizar como referente teórico el IS, resultando en que el eje metodológico es la TFD, ésta se originó en 1960 donde Glasser y Strauss publicaron un libro en 1967 dedicado al desarrollo de una teoría con el fin de explicar fenómenos sociales denominado "El descubrimiento de la teoría fundamentada: estrategias para la investigación cualitativa ${ }^{4}$.

Glaser y Strauss referían que las teorías que se desarrollaban debían comprobarse, por lo que propusieron una metodología en la que la teoría surgiera de los datos, para lo cual, el investigador debía abordar el estudio de manera inductiva, libre de preconceptos a probar o refutar, para con ello lograr descubrir la principal preocupación de los participantes, por lo que también el investigador debe desconocer a qué conducirá la investigación?

Cabe señalar que Glasser y Strauss diseñaron una metodología en la que el análisis de los datos, así como su colecta, debe darse de manera simultánea, para su desarrollo se utiliza el muestreo teórico, la codificación ya sea selectiva, axial o abierta, el Método de Comparación Constante (MCC) y la redacción de memos.

En la actualidad se describen tres corrientes, éstas son la Teoría Fundamentada Clásica (TFC) por Barney Glaser, la Teoría Fundamentada Modificada (TFM), transformada o Straussiana y la Teoría Fundamentada constructivista (TFCn) planteada por Charmaz en donde de manera general estas corrientes presentan similitudes referentes con el método original en aspectos como el uso del muestreo teórico, saturación de datos, memos, un análisis comparativo constante, entre otros ${ }^{7-8}$.

De acuerdo con la corriente filosófica de donde surge la TFD, Estrada refiere que la TFM surge del interaccionismo simbólico y de la fenomenología en la que se estudian las interacciones y WWestructuras de una realidad social, así como el significado de las experiencias como parte del interaccionismo simbólico.

Por su parte la TFCn surge del Constructivismo Social en la que el aprendizaje y la investigación forman parte de la vida social. Por último, se plantea que el Realismo Crítico es la corriente de pensamiento de la TFC en la que se construye la teoría para conocer el abordaje con relación a las soluciones del fenómeno que se analiza ${ }^{8}$. 


\section{Las bases metodológicas de la Teoría Fundamentada en los Datos}

La TFD utiliza la herramienta de Método de Comparación Constante para encontrar las similitudes y los contrastes de los datos identificando sus relaciones, características y lo que determina esas relaciones ${ }^{9}$.

El MCC permite identificar las categorías teóricas, por lo que a través de las entrevistas que se aplican y la observación, se hará una comparación para obtener los temas esenciales y comprender el fenómeno de estudio ${ }^{6}$.

Asimismo, cabe señalar que la TFD utiliza el muestreo teórico, el cual se basa en seleccionar a participantes conforme a la necesidad de refinar y precisar la teoría en desarrollo ${ }^{10}$. Es decir, la colecta de los datos se guía acorde a los conceptos que se derivan de la teoría que se encuentra en construcción a través de la comparación constante, por lo que el muestreo teórico permite añadir a participantes para lograr una mejor comprensión de la información y una mayor densidad de las categorías.

Además, se utiliza la saturación teórica considerando que se cumple cuando todas las categorías están saturadas, refiriéndose a que no existen datos que permitan emerger una nueva categoría, reafirmando que, las categorías construidas ya se encuentran completamente desarrolladas, así como sus relaciones.

Es fundamental mencionar que la saturación teórica es indispensable, pues indica el momento en el cual se debe detener la colecta de datos, dado que no se encuentra alguna información relevante que pudiese explicar las categorías existentes, así como el aportar la creación de una nueva que coadyuve a la comprensión del fenómeno.

Por último, los memos se definen como notas en donde el investigador registra apuntes, reflexiones, dudas, hipótesis, etc., para elaborar relaciones entre los datos, criterios de selección, entre otros, lo que brinda una mayor riqueza de los datos del fenómeno de estudio. Es decir, los memos son notas para considerar conexiones y descubrir la categoría central que ayudará a la propuesta y generación de la teoría.

En la TFD el análisis y la colecta de datos se realiza en secuencias alternadas, es decir, el análisis se da con la primera entrevista y eso lleva a más análisis y a más entrevistas y así sucesivamente, por lo que el análisis es lo que impulsa la colecta de datos. Es importante referir que, para este punto el investigador se encuentra moldeado por los datos y los datos por el investigador, por lo que pueden surgir problemas entre la objetividad y la sensibilidad, en donde la objetividad es fundamental para que pueda desarrollarse una interpretación imparcial y precisa del fenómeno, así como la sensibilidad es esencial para percibir de manera sutil los significados y sus conexiones conceptuales ${ }^{10}$.

Debido a lo anterior, es primordial reconocer la subjetividad y tomar las medidas apropiadas para evitar en lo posible la intromisión en el análisis, así como en los resultados.

\section{Componentes en el proceso de investigación de TFD}


Además, como parte del MCC se utilizan 3 tipos de codificación, la primera es la codificación abierta, ésta divide y codifica los datos en conceptos y categorías, lo que significa desglosar los datos en unidades que puedan brindar un significado. La codificación axial compara los nuevos datos con las categorías anteriores y genera hipótesis cuyo propósito es buscar la relación entre las categorías y subcategorías acorde a las dimensiones y propiedades. Por último, la codificación selectiva integra las categorías para reducir la teoría y define la categoría principal que es la que explica y da sentido a la relación de los datos.

Es relevante señalar que a la par en la que los datos son recopilados, se sugiere codificar, comparar y organizar en categorías cada vez más abstractas para sugerir una teoría basada realmente en los datos obtenidos.

\section{La sexualidad y su comprensión desde la teoría fundamentada}

La sexualidad es definida como un aspecto central del ser humano, el cual está presente en todas las etapas de la vida abarcando aspectos como el sexo, las identidades y los papeles de género, el placer, el erotismo, la intimidad, la reproductividad y la orientación sexual, ésta se vivencia a través de pensamientos, deseos, fantasías, creencias, papeles y las diferentes relaciones interpersonales ${ }^{11}$.

Cabe destacar que, la sexualidad se va construyendo a través de la interacción entre las personas y la estructura social, así como de la interacción de factores biológicos, psicológicos, socioeconómicos, culturales, éticos, religiosos y/o espirituales ${ }^{12}$.

A continuación, se describen algunos de los fenómenos que ha abordado el profesional de enfermería en el tema de sexualidad utilizando como referente metodológico la TFD, para el análisis de la información, emergieron algunas categorías para brindar una mejor comprensión de los fenómenos abordados:

\section{a. Infecciones de transmisión sexual}

Se encontraron dos estudios, en el primero se indagaron las condiciones socioculturales que pueden determinar las altas cifras de infecciones de transmisión sexual sobre las mujeres de piel oscura, con el propósito de desarrollar programas efectivos de prevención y que sean culturalmente aceptables, en éste se evidencia la influencia de los factores socioculturales sobre la autoimagen, las relaciones íntimas y la salud sexual ${ }^{13}$, en el estudio de Rhodes, et al., el objetivo fue identificar y explorar los elementos de participación comunitaria para la prevención, tratamiento y detección de las ITS ${ }^{14}$.

\section{b. Toma de decisiones}

En cuanto a la toma de decisiones en materia de sexualidad se encontraron dos estudios, en el estudio de Ezer, et al., se obtuvo información sobre el proceso de toma de decisiones sexuales de adolescentes, así como factores que influyen en ese proceso de manera positiva y negativa, destacando los valores y circunstancias personales, valores y expectativas familiares, influencias de los amigos y de la comunidad ${ }^{15}$, en el estudio de Konkle en cambio se aplicó para intentar explicar procesos de toma de decisiones en mujeres para determinar el uso de autoprotección durante las actividades sexuales de riesgo en la que se propone desarrollar intervenciones que empoderen a las mujeres y motiven a los hombres a realizarse detecciones oportunas ${ }^{16}$. 
Esto permitió comprender de mejor manera el proceso de toma de decisiones para autoprotección, así como el contexto que influye en ese sentido para orientar el desarrollo de futuras investigaciones.

\section{c. Vacunación y Virus del Papiloma Humano (VPH)}

Se encontraron dos estudios referentes al tema, en ambos se evalúo la comunicación entre la madre y la hija en cuanto a decidir sobre la aplicación de la vacuna contra el VPH en la que se destaca mejorar la comunicación entre madre e hija para disminuir los riesgos en salud, así como la educación en el tema ${ }^{17}$ y una vez que se comprenda el tema y la manera en cómo influye la cultura, se podrán sugerir campañas educativas con más información, consistencia, educación sobre el VPH y propaganda basada en su contexto y cultura ${ }^{18}$.

\section{d. Diversidad}

De la misma manera se han realizado estudios para explorar cómo los hombres homosexuales desarrollan la resiliencia a lo largo de su vida, en especial, se basaron en aquellos procesos externos que la comprenden para promover la salud, entre los hallazgos encontraron la profunda discriminación que esta población enfrenta, por lo que refieren que es significativa la necesidad de brindar servicios en salud a través de profesionales flexibles, que conozcan la problemática y brinden un cuidado basado en el respeto ${ }^{19}$, así como reconocer las vivencias propias del colectivo para ofrecer cuidados basados en sus necesidades y particularidades ${ }^{20}$.

\section{e. Vivencias en sexualidad}

En cuanto al tema de las vivencias en sexualidad, Nascimiento utilizó la TFD para interpretar las vivencias en la mujer mayor y construir un modelo teórico explicativo en donde las categorías que surgieron son la percepción de la modificación del propio envejecimiento y el del compañero, reflexión sobre la permanencia de la sexualidad en el envejecimiento, dificultades sociales y culturales, así como la búsqueda de alternativas para vivir la sexualidad ${ }^{21}$, permitiendo conocer con claridad el comportamiento del fenómeno siendo la sexualidad una experiencia permeada por cambios físicos, dificultades emocionales, sociales y culturales a los que deben adaptarse, así mismo, se evidenció la dificultad de hablar de estos temas por parte de las participantes.

Ramírez, et al., en su estudio buscó conocer las percepciones de mujeres trabajadoras sexuales acerca de su cuerpo por medio de sus vivencias y experiencias, lo que permitió comprender la importancia de la construcción social del cuerpo considerando el ambiente del lugar de trabajo, la imagen social que proyectan, así como sus experiencias, determinarán los cuidados que enfermería otorgará según las necesidades y potencialidades de estas mujeres ${ }^{22}$.

Zambrano propone un planteamiento teórico en el que describe cómo la población adolescente y joven interioriza símbolos y visiones que estructuran los imaginarios en sexualidad, lo que permite analizar la visión de los jóvenes desde los diferentes abordajes que recibe ${ }^{23}$.

\section{f. Retos en el cuidado de la sexualidad}


Rizkalla en su estudio sobre la violencia de pareja propone dilucidar las barreras y facilitadores de brindar atención en mujeres que experimentan violencia y recomendar estrategias, por lo que refiere que los profesionales de salud a menudo experimentan falta de capacitación para abordar el tema, así como un lugar al que se deriven los casos y el temor de poner en riesgo la relación paciente - proveedor $^{24}$.

Rodolfo, et al., realizaron la colecta de datos en personal enfermero con el objetivo de discutir estrategias que funjan como parte del desarrollo saludable en el adolescente, refiriendo que la complejidad que se experimenta en el ejercicio de la sexualidad indica la necesidad de elaborar estrategias individuales y basadas en el contexto, así como fenómenos como el abuso y la diversidad sexuales asegurando su acceso a los servicios de salud y una atención adecuada ${ }^{25}$.

Valenzuela propone conocer la percepción que tiene el profesional de la salud sobre la valoración en sexualidad de los adultos mayores, encontrando que los profesionales refieren la existencia de factores como los valores personales, estructurales, sociales y culturales, así como el estigma, falta de conocimiento en sexualidad y su abordaje como dificultades para tratar el tema con el usuario, por lo que perciben la falta de formación específica en el tema ${ }^{26}$.

\section{g. Salud materno infantil}

En el estudio desarrollado por Muwanguzi, et al., exploraron el significado de la participación de la pareja hombre, así como proponer una definición y modelo teórico de este concepto, por lo que es importante desempeñar actividades de sensibilización sobre la importancia de la participación del hombre en la salud materno infantil ${ }^{27}$.

\section{h. Género y cuidado}

En cuanto al género y cuidado se realizó un estudio para analizar las experiencias de hombres y mujeres respecto a la cirugía de revascularización miocárdica desde una perspectiva de género, encontrando que existe una diferencia significativa entre sexos en cuanto a la categoría: percibir la sexualidad después de la cirugía, refieren disminución en la actividad sexual y dificultad para abordar el tema, reconociendo la importancia de considerar la perspectiva de género en la gestión de cuidados con el fin de planear mejores cuidados $^{28}$.

Respecto al estudio de Botello y Casado plantearon el objetivo de conocer los estereotipos relacionados con la menarquia y la menopausia así como las implicaciones para la salud, en éste descubrieron que la menarquia se asocia a que la niña se ha convertido en mujer sin contemplar otra madurez como la emocional y psicológica, respecto a la menopausia descubrieron connotaciones negativas, en la que la mujer es percibida como una mujer vieja causando depresión y temor a quienes viven la etapa, así como a las que se dirigen a ella, por lo que se propone erradicar los prejuicios culturales a través de la educación ${ }^{29}$.

Es importante señalar que la sexualidad es un aspecto cada vez más incluido en el estudio, sin embargo, por la propia naturaleza de ser una construcción social, carece de ser estudiada y abordada por parte del usuario y del profesional de enfermería como parte del cuidado para lograr el bienestar integral, así mismo, estos estudios permiten identificar las necesidades en atención, profundizar de mayor manera en el fenómeno, así como la relevancia de brindar un cuidado basado en el contexto e individualidad. 


\section{Conclusiones}

Enfermería como ciencia y disciplina debe considerar todos los aspectos relevantes que afectan la salud de las personas, por lo que es importante continuar con la investigación cualitativa para indagar otros aspectos de los fenómenos que aquejan el bienestar de las personas y así generar innovaciones en los conocimientos para mejorar la calidad en los cuidados que proporciona enfermería.

Al generar nuevos conocimientos, se obtendrán nuevos horizontes y caminos metodológicos para mejorar el cuidado, dado que el compromiso profesional de enfermería es estar en constante transformación para responder a un deber ético-moral con las personas.

La investigación cualitativa es evidentemente un enfoque que permite describir, comprender y develar fenómenos que no pueden ser reducidos a números, debido a que, a través de las diversas técnicas y métodos de colecta de datos se obtiene la experiencia y el significado de quienes lo viven, lo que permite obtener datos valiosos de las realidades que necesitan ser comprendidas.

Por su parte, la TFD es un método que exige ser contemplada para el desarrollo de investigación en enfermería, pues siendo una profesión cuya razón de ser es el cuidado, requiere de la cercanía y comprensión del otro, de develar significados propios de cada participante que, si bien los resultados no pueden ser generalizados, la propuesta de una nueva teoría y una mayor profundidad del fenómeno permitirá el desarrollo de exitosos modelos de cuidado por el progreso de estrategias basadas en los datos obtenidos previamente.

La TFD ofrece otro aspecto de comprensión de los mundos sociales y las interacciones que han sido significativas en la vida de las personas que representan su actual manera de actuar, por lo que la construcción de las categorías proporciona voz a esas experiencias vividas sentando las bases científicas para futuras investigaciones, cambios en la práctica, adecuaciones, generación de políticas y cuidados que impacten de manera positiva y trascendental en la vida de las personas.

Por lo que, la aplicación de la TFD dentro del área de la sexualidad es de suma importancia, pues ésta es construida a través de la interacción con el contexto, las personas, la etapa de desarrollo, entre otras, lo que exige la necesidad de conocer las distintas carencias en atención de las diversas poblaciones para brindar un cuidado integral. Por ejemplo, en cuanto a las ITS es significativo que el profesional considere las condiciones socioculturales que influyen para su incidencia o prevención, así como proponer intervenciones acordes a ese contexto y que sean aceptables socialmente, en referencia a la toma de decisiones en sexualidad, debe trabajarse en la motivación y empoderamiento para lograr el asertividad sexual y con ello disminuir los riesgos en salud.

En cuanto a la vacunación y el VPH, se debe fomentar y reforzar la comunicación con la familia, así como la educación en salud para su prevención, lo que también puede considerarse para temas de diversidad, pues se debe indagar sobre su inclusión y otorgar un cuidado con base en las necesidades particulares de la población. Ahora, con relación a las vivencias en sexualidad, es fundamental que se consideren los cambios que surgen en cada etapa, así como las diferencias que se experimentan, esto permitirá que el profesional pueda brindar una atención en sexualidad acorde a las características particulares de cada individuo. 
Por tal motivo, el profesional de la salud debe estar en constante capacitación y actualización para ofrecer un cuidado de calidad frente a los distintos retos existentes sobre el fenómeno de la sexualidad, pues los resultados de la búsqueda de información científica, evidencian la falta de formación especial por parte del profesional de enfermería para abordar la atención en sexualidad, pues el mismo profesional refiere falta de capacitación que oriente la práctica, así como la falta de estrategias que promuevan la inclusión de la diversa población y sus necesidades.

Ante los argumentos mencionados, es importante referir que existe poca apertura por parte de la persona, lo que limita brindar atención en sexualidad, pues de la misma manera es un tema que resulta incómodo de abordar por parte de algunos usuarios limitándose a hacer cuestiones en el tema generando una brecha entre quien brinda la atención como por parte de quien la recibe.

Por último, es trascendental enfatizar que enfermería desarrolle una formación integral en la que contemple los diferentes enfoques de la investigación y así seguir consolidando su propio cuerpo de conocimientos, e incluir en su atención las distintas dimensiones que componen la sexualidad como el autoconocimiento, las relaciones afectivas, y así no solo enfocar su atención en la prevención de las infecciones de transmisión sexual y los embarazos no intencionados.

\section{Referencias}

1. Osorio-Castaño JH. La salud sexual y reproductiva como área para desarrollar la enfermería de práctica avanzada. Rev. Cienc. Cuidad [Internet]. Mayo de 2017 [Consultado 18 May 2021]; 14(2): 130-43. Disponible en: https://dialnet.unirioja.es/servlet/articulo?codigo=6052714

2. Hernández-Sampieri R, Mendoza-Torres CP. Metodología de la investigación: las rutas cuantitativa, cualitativa y mixta. Ciudad de México: D.F: McGraw-Hill Interamericana; 2018, 4-21 p.

3. Natera-Gutiérrez SI, Guerrero-Castañeda RF, Ledesma-Delgado ME, Ojeda-Vargas MG. Interaccionismo simbólico y teoría fundamentada: un camino para enfermería para comprender los significados. Cult cuid [Internet]. Mayo de 2017 [Consultado 05 May 2021]; 21(49): 190-9. Disponible en: https://rua.ua.es/dspace/bitstream/10045/72942/1/CultCuid_49_21.pdf

4. Oliveira N, Sodré B, Ribeiro M. Teoría fundamentada en datos: aspectos metodológicos en la tesis de enfermería brasileña. Rev. Bras. Enferm [Internet]. Febrero de 2020 [Consultado 16 May 2021]; 73(6): 1 - 8. Disponible en: http://dx.doi.org/10.1590/0034-7167-2019-0274

5. Castro M, Simian D. La enfermería y la investigación. Rev Med Clin Condes [Internet]. Junio de 2018 [Consultado 18 May 2021]; 29(3): 301-10. Disponible en: https://doi.org/10.1016/j.rmclc.2018.04.007

6. Vivar C, Arantzamendi M, López-Dicastillo O, Gordo C. La teoría fundamentada como metodología de investigación cualitativa en enfermería. Index Enferm [Internet]. Diciembre de 2010 [Consultado 12 May 2021]; 19(4). Disponible http://scielo.isciii.es/scielo.php?script=sci_arttext\&pid=S1132-12962010000300011\&lng=es.

7. Kenny M, Fourie R. Tracing the history of grounded theory methodology: from formation to fragmentation. TQR [Internet]. Diciembre de 2014 [Consultado 18 May 2021]; 19 (52): 1-9. Disponible en: http://www.nova.edu/ssss/QR/QR19/kenny103.pdf

8. Estrada-Acuña R, Giraldo C, Arzuaga M. Aproximación al análisis de datos cualitativos en Teoría Fundamentada desde la perspectiva clásica. Relmis.com.ar [Internet]. Octubre de 2020 [Consultado 13 May 2021]; 20(10): 19-37. Disponible en: http://relmis.com.ar/ojs/index.php/relmis/article/view/aproximacion_al_analisis/168

9. González L. XVII Jornadas de Investigación de la Facultad de Ciencias Sociales UdelaR, 2018. Experiencias de acoso sexual callejero: miradas desde el interaccionismo simbólico [Internet]. Uruguay: Universidad República de Uruguay; 2018 [Consultado 19 May 2021]; 1-23

10. Strauss A, Corbin J. Bases de la investigación cualitativa. Técnicas y procedimientos para desarrollar la teoría fundamentada. Colombia: Editorial Universidad de Antioquia; 2002.1-45 p. 
11. World Association for Sexual Health. Salud sexual para el Milenio Declaración y documento técnico [Internet]. Washington: OPS; 2009 [Consultado 12 May 2021]. Disponible en https://www.paho.org/hq/dmdocuments/2010/Salud\%20Sexual\%20para\%20el\%20Milenio.pdf

12. Secretaría de salud. Programa de acción específico Salud sexual y reproductiva para adolescentes 2013-2018 Programa sectorial de salud [Internet]. Secretaría de Salud, 2013 [Consultado 12 May 2021]. Disponible

en: https://www.gob.mx/cms/uploads/attachment/file/242381/SaludSexualyReproductivaparaAdolesc entes_2013_2018.pdf

13. Crooks N, King B, Tluczek A, McDermott J. The process of becoming a sexual black woman: a grounded theory study. Perspect sex reprod health [Internet]. Enero de 2019 [Consultado 14 May 2021]; 51(1): 17-25. Disponible en: https://onlinelibrary.wiley.com/doi/abs/10.1363/psrh.12085

14. Rhodes S, Daniel-Ulloa J, Wright S, Mann-Jackson L, Johnson D, Hayes N, et al. Critical elements of community engagement to address disparities and related social determinants of health: the centers of disease control and prevention community approaches to reducing sexually transmitted disease iniciative. Sex transm dis [Internet]. Enero de 2021 [Consultado 05 May 2021]; 48(1): 4955. Disponible en: https://www.ncbi.nlm.nih.gov/pmc/articles/PMC7736098/

15. Ezer $P$, Leipert $B$, Evans M, Regan S. Toma de decisiones de adolescentes heterosexuales sobre las relaciones sexuales y el embarazo en las zonas rurales de Ontario, Canadá. Salud rural y remota [Internet]. Enero de 2016 [Consultado 08 May 2021]; 16: 3664. Disponible en: https://www.rrh.org.au/journal/article/3664

16. Konkle-Parker D, Fouquier K, Portz K, Wheeless L, Arnold T, Harris C. et al. Women's decisiónmaking about self-protection during sexual activity in the deep south of the USA: a grounded theory study. Cult health sex [Internet]. Enero de 2018 [Consultado 08 May 2021]; 20(1): 84-9. Disponible en: https://pubmed.ncbi.nlm.nih.gov/28621176/

17. Colón-López V, Fernández-Espada N, Vélez C, Gonzalez V, Díaz-Toro E, Calo WA. et al. Communication about sex and HPV among Puerto Rican mothers and daughters. Ethn health [Internet]. Agosto de 2017 [Consultado 08 May 2021]; 22(4): 348-60. Disponible en: https://pubmed.ncbi.nlm.nih.gov/27882783/

18. Galbraith-Gyan K, Lechuga J, Jenerette C, Palmer M, Moore A, Hamilton J. HPV vaccine acceptance among African-American mothers and their daughters: an inquiry grounded in culture. Ethn health [Internet]. Mayo de 2017 [Consultado 12 May 2021]; 24(3): 323-40. Disponible en: https://pubmed.ncbi.nlm.nih.gov/28553758/

19. Handlovsky I, Bungay V, Oliffe J, Johnson J. Developing resilience: gay men's response to systemic discrimination. Am J mens health [Internet]. Septiembre de 2018 [Consultado 12 May 2021]; 12(5): 1473-85. Disponible en: https://pubmed.ncbi.nlm.nih.gov/29683025/

20. Galindo S, Gómez P, Manosalva M. Momentos de reconocimiento como sujetos de derecho de un grupo de personas jóvenes universitarias LGBT en Tunja, Boyacá. Sex Salud Soc [Internet]. Agosto de 2018 [Consultado 12 May 2021]; (29): 172-94. Disponible en: https://www.researchgate.net/publication/328417360_Momentos_de_reconocimiento_como_suje tos_de_derecho_de_un_grupo_de_personas_jovenes_universitarias_LGBT_en_Tunja_Boyaca

21. Fernandes R, Sanches M, Moreira S, Ribeiro M. [Experiencia de la sexualidad pr señoras mayores]. Rev enferm UER] [Internet]. Agosto de 2017 [Consultado 11 May 2021]; 25: [e20892]. Disponible en: $\quad$ https://www.e-publicacoes.uerj.br/index.php/enfermagemuerj/article/view/20892/22650 Portugués

22. Ramírez M, Casis D, Castellano M, Covarrubias A, Figueroa D, López J, et al. Percepciones corporales en trabajadoras sexuales. Enfermería: Cuidados Humanizados [Internet]. Junio de 2017 [Consultado 11 May 2021]; 6(1): 37-45. Disponible en: https://revistas.ucu.edu.uy/index.php/enfermeriacuidadoshumanizados/article/view/1367

23. Zambrano-Plata G, Bautista-Rodríguez L, López V. Imaginarios de sexualidad en estudiantes universitarios. Rev. salud pública [Internet]. Julio de 2018 [Consultado 11 May 2021]; 20(4): 408. Disponible en: https://revistas.unal.edu.co/index.php/revsaludpublica/article/view/52320

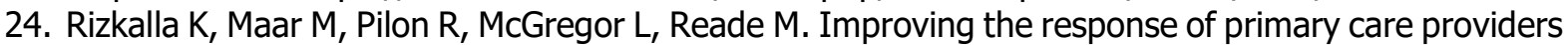
to rural First Nation women who experience intimate partner violence: a qualitative study. BMC Womens Health [Internet]. Septiembre de 2020 [Consultado 08 May 2021]; 20(1): 209. Disponible en: https://pubmed.ncbi.nlm.nih.gov/32957935/ 
25. Rodolfo I, Privado T, Sá Basílio S, Carvalho M, Luzia J. [Órdenes y desórdenes- complejidad del inicio de la adolescencia y la salud sexual: contribuciones para la enfermería]. Rev Enferm UERJ [Internet]. Enero de 2016 [Consultado 08 May 2021]; 24(2): e4569. Disponible en: https://www.epublicacoes.uerj.br/index.php/enfermagemuerj/article/view/14569/19554 Portugués

26. Valenzuela D, Torres A, Mellado M, Palominos J, Quijada D, García G. Percepción de profesionales de enfermería sobre la valoración de la sexualidad en adultos mayores (Chile). Cult cuid [Internet]. Noviembre de 2019 [Consultado 12 May 2021]; 23(55): 68-77. Disponible en: https://rua.ua.es/dspace/bitstream/10045/100852/1/CultCuid_55-68-77.pdf

27. Muwanguzi $P$, Nassuna L, Voss J, Kigozi J, Muganzi A, Denis T, et al. Towards a definition of male partner involvement in the prevention of mother -to- child transmission of HIV in Uganda: a pragmatic grounded theory approach. BMC health serv res [Internet]. Agosto de 2019 [Consultado 16 May 2021]; 19: 557.2 Disponible https://bmchealthservres.biomedcentral.com/articles/10.1186/s12913-019-4401-x

28. Pedroso M. Melo G, Koerich C, Callegaro G, Aparecida M, Lorenzini A. [El proceso de vivir la cirugía de revascularización cardiaca: un análisis de género]. Esc. Anna Nery [Internet]. Diciembre de 2016 [Consultado 18 May 2021]; 20(4): e20160093. Disponible en: https://www.redalyc.org/pdf/1277/127746815013.pdf Portugués

29. Botello A, Casado R. Estereotipos de género con respecto a las etapas reproductivas de las mujeres y sus implicaciones en la salud. Matronas prof [Internet]. Abril de 2016 [Consultado 16 May 2021]; 17(4): 130-6. Disponible en: https://www.federacion-matronas.org/revista/wpcontent/uploads/2018/01/original-estereotipos-de-genero.pdf

\section{Editor}

Escuela de Enfermería de la Universidad Católica Santo Toribio de Mogrovejo, Chiclayo, Perú

\section{Cómo citar este trabajo}

Cervantes-Lara C, Casique-Casique L, Álvarez-Aguirre A. Teoría fundamentada como referente metodológico en el cuidado de enfermería. Acc Cietna: para el cuidado de la salud [Internet]. 2021; 8(2): 95 - 105. Disponible en: https://doi.org/10.35383/cietna.v8i2.688

\section{Financiación}

El presente artículo no cuenta con financiación específica de agencias de financiamiento en los sectores público o privado para su desarrollo y/o publicación.

\section{Conflicto de interés}

Los autores del artículo declaran no tener ningún conflicto de intereses en su realización.

C Los autores. Este artículo es publicado por la Revista Acc Cietna: para el cuidado de la salud de la Escuela de Enfermería, Universidad Católica Santo Toribio de Mogrovejo. Este es un artículo de acceso abierto, distribuido bajo los términos de la Licencia Creative Commons Atribución-NoComercial-CompartirIgual 4.0 Internacional (CC BY-NC-SA 4.0), que permite el uso no comercial, distribución y reproducción en cualquier medio, siempre que la obra original sea debidamente citada. 